\title{
Floating Point versus Fixed point Tradeoffs in FPGA Implementations of QR Decomposition Algorithm
}

\author{
Siavash Amin-Nejad, Katayoon Basharkhah, Tayyebeh Asgari Gashteroodkhani
}

\begin{abstract}
A wide variety of digital communication systems are encountered with high computational tasks. QR decomposition is one of such algorithms that can be implemented on FPGAs as a solution to large complex matrix inversion problems. A flexible vector processing architecture for the fixed and floating point implementations of the $Q R$ decomposition is presented. The design is implemented on the StratixIV device with $230 \mathrm{~K}$ logic elements and verified with the SignalTap II built-in logic analyzer. Throughputs of $2.4 \mathrm{M}$ and 2.11M decompositions per second with maximum clock frequency of $340 \mathrm{MHz}$ and $360 \mathrm{MHz}$ are achieved for $4 \times 4$ matrices with the fixed and floating point designs respectively. The FPGA resource utilizations of the two data type implementations are also compared for different matrix sizes for the StratixIV and Arria10 devices.
\end{abstract}

Index Terms-QR decomposition, FPGA, Fixed point, Floating Point.

\section{INTRODUCTION}

The advent of new advanced digital communication systems and military radar applications such as adaptive beamforming [1], Space-Time Adaptive Processing, MIMO systems, etc. has imposed complex and computationally demanding algorithms [2]. These algorithms involve matrix inversions or least square minimizations at large sizes and high data rates. Matrix factorization techniques such as LU factorization, Cholesky factorization and QR decomposition are crucial solutions to these problems and many attempts have been made on their hardware realization in the last decades [3, 4]. QR decomposition is widely applied nearly in every field where a numerically stable and robust inversion of large and dense matrices is needed [5]. It involves two prerequisites of least square estimates namely, triangularization and orthogonalization, means that it factorize a matrix A into the product of a unitary quadrature matrix, $\mathrm{Q}$, and an upper triangular matrix, $\mathrm{R}$.

There are three main algorithms for $\mathrm{QR}$ decomposition including Gram -Schmidt and modified Gram-Schmidt, Householder reflections and Givens rotations [6]. Householder transformations need to process in columns and rows so there must be a parallel access across memories. This can be a disadvantage in high speed processing [6]. Previous attempts aimed at Givens Rotation implementation suffer from high latency due to more complex operations [7]. GramSchmidt has the same level of performance in terms of stability and accuracy but requires fewer operations which make it more attractive for both fixed and floating point

Siavash Amin-Nejad is with University of Guilan, (e-mail Siavash.Amin-Nejad@liverpool.ac.uk).

Katayoon Basharkhah was with University of Guilan, Rasht, Iran. She is now with the Department of Electrical Engineering, University of Tehran, Iran (e-mail: ktbasharkhah@gmail.com). calculations. Applying slight modifications to the GramSchmidt algorithm gives the modified Gram-Schmidt (MGS) algorithm which has better accuracy than the classic one [8].

Due to the extensive computational burden of the QR decomposition, ASICs and FPGAs are two candidates for the hardware implementation. ASICs serves best in the low power and high speed computation requirements in the large volumes of production. On the other hand, FPGAs with powerful and abundant DSP Blocks, RAM Blocks and adaptive LUTs offer flexibility, parallelism and high throughput at much lower cost [9-10]. FPGAs with short time to market are suitable for mid volume productions but suffer from more power consumption and less clock frequency.

Most of the time FPGAs are used for fixed point rather than floating point designs because the latter suffers from long data path latencies, routing congestion and reduced Fmax. Fused data path can be an alternative method for floating point implementation that provides hardware friendly representation and also reduced redundancies [6], [11]. In this paper we propose a flexible architecture designed for both fixed and floating point implementation using fused data path. In section II the QR Decomposition algorithm as a parameterizable core for different matrix sizes and different vector sizes using vector processing method is described. The proposed architecture is described in section II. Section IV devotes to explaining fixed and floating point representations. The hardware implementation of the design on the Altera's DE4 development board with StratixIV FPGA is covered in section V. Performance analysis and comparisons are presented in section VI. Conclusion and discussions are given in section VII.

There are many ways to solve the linear system equation $A x=B$ to find the unknown vector [12]. In analytic approach which uses determinants, matrix inversion can be completely a bottleneck as it needs too many complicated operations and results in non-scalable architectures [13]. This necessitates the usage of decomposition methods for the solution of the large size matrices. QR decomposition is one of these techniques that can be used for any general $\mathrm{m}$ by $\mathrm{n}$ matrix unlike other methods such as Cholesky solver which are applied only on square and symmetric matrices [6]. The result of the $\mathrm{QR}$ decomposition for a $[\mathrm{m} \times \mathrm{n}]$ matrix, $\mathrm{A}$, will be an orthonormal $[\mathrm{m} \times \mathrm{n}]$ matrix, $\mathrm{Q}$, and an upper triangular $[\mathrm{n} \times \mathrm{n}]$ matrix, R. Replacing A with the new decomposed matrices $Q$ and $\mathrm{R}$ results in $Q R x=B$. Multiplying both sides from left by $Q^{T}$ and considering $Q^{T} Q=I$ results in $R x=Q^{T} B$. Now

Tayyebeh Asgari Gashteroodkhani is with the Electrical Engineering Department, University of Guilan, Rasht, Iran

(e-mail: tayyebeh.asgari19@yahoo.com). 
can be calculated by the Backward Substitution method without needing for the matrix inversion.

\section{QR DECOMPOSITION USING MODIFIED GRAM-SCHMIDT}

Projection of vector (a) over vector (q) in an $\mathrm{m}$ dimensional space is defined as:

$$
\operatorname{proj}_{q}(a)=\frac{\langle a . q\rangle}{\langle q . q\rangle} q
$$

Where $<\mathrm{q} . \mathrm{a}>$ represents the inner product.

In the following equations $\mathrm{u}_{1}, \mathrm{u}_{2} \ldots \mathrm{u}_{\mathrm{n}}$ are orthogonal and $\mathrm{q}_{1}, \mathrm{q}_{2} \ldots \mathrm{q}_{\mathrm{n}}$ are their corresponding orthonormal set.

$$
\begin{aligned}
& u_{1}=a_{1} \quad, \quad q_{1}=\frac{u_{1}}{\left\|u_{1}\right\|} \\
& u_{2}=a_{2}-\operatorname{proj}_{q_{1}}\left(a_{2}\right) \quad, \quad q_{2}=\frac{u_{2}}{\left\|u_{2}\right\|} \\
& u_{n}=a_{n}-\sum_{j=1}^{n} \operatorname{proj}_{q_{j}}\left(a_{n}\right) \quad, \quad q_{n}=\frac{u_{n}}{\left\|u_{n}\right\|}
\end{aligned}
$$

After rearranging the equations:

$$
\begin{aligned}
& a_{1}=q_{1} \cdot\left\|u_{1}\right\| \\
& a_{2}=\operatorname{proj}_{q_{1}}\left(a_{2}\right)+q_{2} \cdot\left\|u_{2}\right\| \\
& a_{n}=\sum_{j=1}^{n-1} \operatorname{proj}_{q_{j}}\left(a_{n}\right)+q_{n} \cdot\left\|u_{n}\right\|
\end{aligned}
$$

Since qi are unit vectors, equation (3) can be rewritten as: $a_{1}=q_{1} \cdot\left\|u_{1}\right\|$

$$
\begin{aligned}
& a_{2}=<q_{1} \cdot a_{2}>q_{1}+q_{2} \cdot\left\|u_{2}\right\| \\
& a_{n}=\sum_{j=1}^{n-1}<q_{j} \cdot a_{n}>+q_{n} \cdot\left\|u_{n}\right\|
\end{aligned}
$$

And in matrix form:

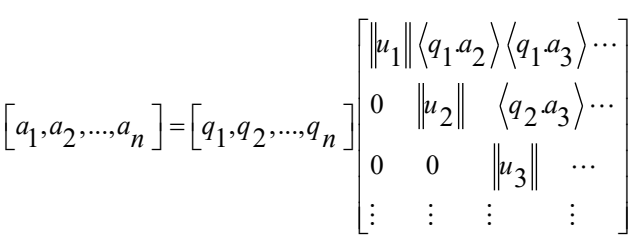

This algorithm can be realized in Matlab by the code in Appendix 2. This algorithm is composed of the processing elements to do norm, which requires $\mathrm{n}$ square root operations and $\mathrm{nm}$ multiplications and (nm-1) additions. It also requires $n(n-1) / 2$ inner products and $n(n-1) / 2$ divisions of vector by scalar. Updates of matrix A requires $m n(n-1) / 2$ multiplications and additions [6], [14].

The overall goal is to design a low-latency high-throughput hardware for this iterative algorithm. Thus any part of the calculation that doesn't need to be repeated for each new piece of data is removed from the inner loop. As it is evident from the algorithm the diagonal elements of the $\mathrm{R}$ matrix must be computed first and then the rest of the R and Q matrices' elements are calculated. The disadvantage of this code is that the elements of Q cannot be calculated unless the update of matrix A is completed first. This can cause long latencies and the system will be halted for some time. The total number of inactive clock cycles is where and are latency of the calculation respectively [14]. In order to remove these latencies the algorithm is modified as shown in Appendix 1:

\section{ARCHITECTURE OF THE PROPOSED IMPLEMENTATION}

Based on the modified Matlab code in the previous section, the calculations are done in the two main for loops and are column-wise. In the first for loop the square of the diagonal element of each column of matrix $\mathrm{R}$ is calculated first and then the non-diagonal elements of its corresponding row are calculated. These row elements are divided to their corresponding diagonal element in next main for loop to generate the correct row elements of matrix R. Before repeating this procedure for the next column the matrix $\mathrm{A}$ elements at the right side of the current column must be updated according to the first for loop code. The Q matrix elements are calculated in second main for loop based on the modified matrix A elements.

Fig. 1 shows the block diagram of the proposed design. The main units are control unit, memory blocks and vector processing unit. The core memory block initially stores matrix A for decomposition. The process is done column by column. Once the process for one column is completed and it is no longer used, the updated version of matrix A will be overwritten in the memory. This provides an optimized design in terms of memory usage. Since the column to be processed $\mathrm{A}(:, \mathrm{k})$ will be used several times for different operations such as dot product and update calculations it is stored in another small and fast memory called circular buffer.

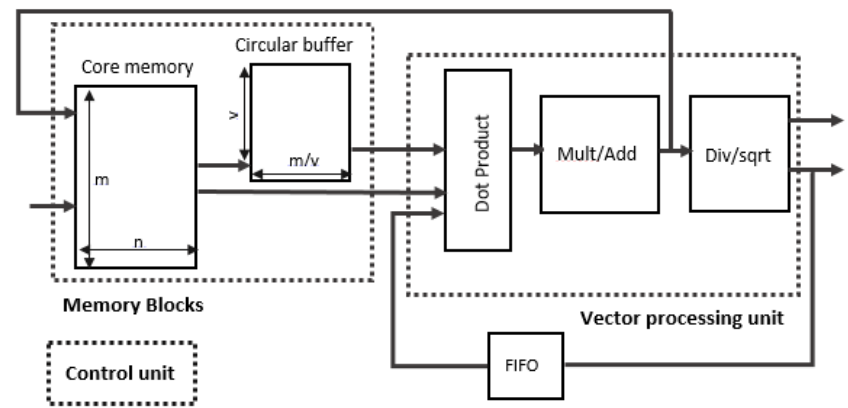

Fig. 1. QRD Block diagram.

Vector processing engine can be considered as the main unit of the design which consists of three subunits: Dot product, Mult/Add and Div/sqrt. Dot product is the major arithmetic operation in this algorithm. The parallel implementation of this operation for large matrix sizes, which is requisite for many digital communication systems, needs a large number of DSP blocks. In order to reduce the number of the DSP blocks in the design, the dot product operation is divided into smaller sizes dot operations which are done sequentially. A compilation design parameter named vector size defines the dot operation size. For the dot operation of two columns of size $m$ and vector size of $v, v$ multipliers are implemented in each clock cycle and $\mathrm{m} / \mathrm{v}$ clock cycles are needed. As shown in Fig. 2, the Dot product unit calculates the vector size dot operation and a single accumulator in Mult/Add unit performs the summation of the results of $\mathrm{m} / \mathrm{v}$ dot operations.

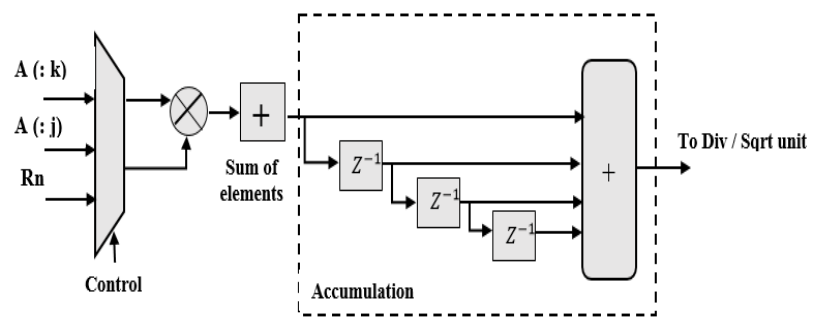

Fig. 2. Dot product block diagram 
As shown in the Matlab code, the second main for loop includes square root and division functions. These functions are calculated in the Div/Sqrt unit which is shown in Fig. 3. The square root function is not available in the DSP Builder library, instead the inverse square root is available which can be implemented by using 3-4 times the required resources for a floating point adder or multiplier and produces one result per clock cycle[6].

The timing and latencies for these iterations will be realized by four nested loop in control unit which serves as a single finite state machine (FSM). The nested loops are attributed to four state in calculations, square calculation, dot product, delay to estimate $\mathrm{R}(\mathrm{k}, \mathrm{k})$ and new updates.

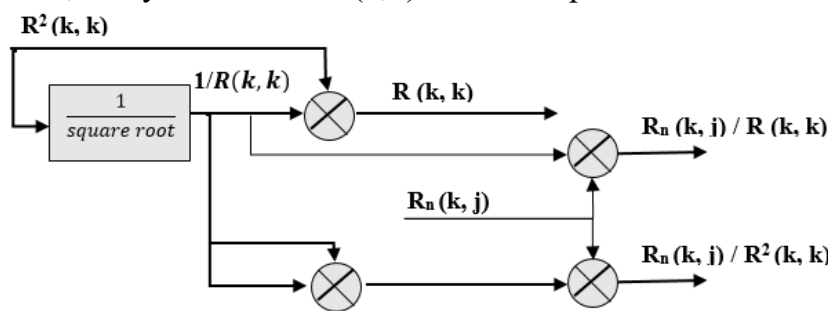

Fig. 4. Sqrt /Division unit block diagram

Fig. 4 shows the timing diagram of the proposed architecture which was obtained from the design simulation. The sequence of operations are also included in this figure. The control unit will produce 8 control signal to provide the required latencies for each step calculations. Signal "Mag" will be active $\mathrm{m} / \mathrm{v}$ cycles to calculate the square of $\mathrm{R}$. In the last cycle signal "Last Mag" will be active to determine when the dot product $(\mathrm{Rn})$ calculation should begin as it comes exactly after R2 and also Div/sqrt unit can calculate the square root and it's inverse. When column $\mathrm{k}$ is under processing, the dot product must be operated on the remained columns so $(\mathrm{n}-\mathrm{k})^{*} \mathrm{~m} / \mathrm{v}$ cycle is needed after R2. After dot product, $\mathrm{Rn} / \mathrm{r} \mathrm{kk}$ and $\mathrm{Rn} / \mathrm{r} \mathrm{kk} 2$ values are prepared and $\mathrm{sub}$ operation cannot be complemented unless this task is done. So a control signal named "check_rkk" alerts to wait for these values and once it is completed signal rkk_valid will have a token. This is the time that sub operation begins. It also needs $(\mathrm{n}-\mathrm{k})^{*} \mathrm{~m} / \mathrm{v}$ cycles. During this time updated value of A are estimated and $\mathrm{Q}$ values can be calculated after A values are ready. This figure also shows the memory blocks' read or write mode.

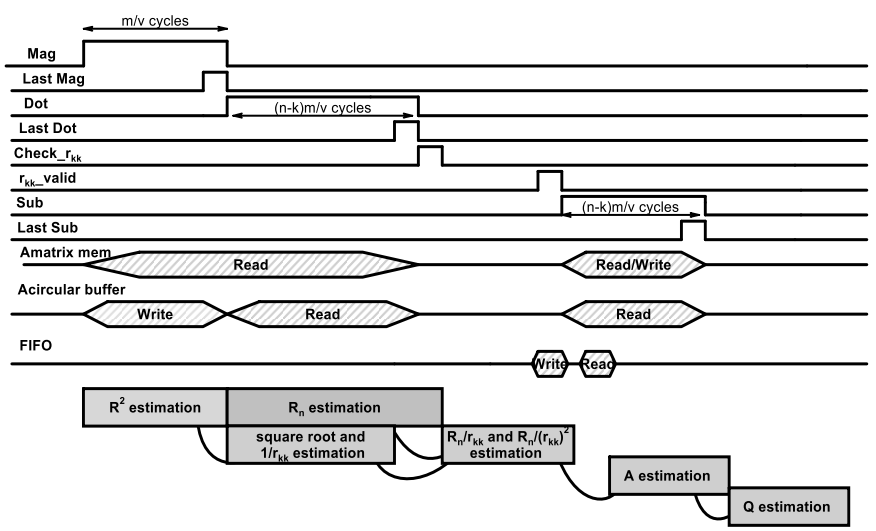

Fig. 4. control signals and operation sequences

\section{FIXED AND FLOATING POINT ARITHMETIC}

Real numbers are presented in two representation system, fixed point and floating point. The two's complement fixed point representation consists of three part, sign bit, integer bits and fractional bits. The number of integer bits defines the highest absolute number and the number of the fraction bits defines precision [15]. The dynamic range of a fixed point number is determined by its word length [2word length -1 ]. This shows a dynamic range of approximately 6 times the word length which is considered so small for some digital processing systems like military applications. The finite word nature of fixed point numbers introduces truncation error and there may be rounding errors too. However the hardware efficient implementation and lower latency of fixed point systems make them an attractive option for $Q R$ decomposition. On the other hand, floating point numbers can provide much more dynamic range and higher accuracy at the expense of long processing. As it is evident, the tradeoff between hardware efficiency, latency and accuracy will affect the choice between fixed or floating point designs.

According to IEEE754 standard the 32-bit single precision floating point format is represented using one sign bit, 8 exponent bits and 23 mantissa bits as follows:

Floating point value $=(-1)^{\text {sign }} \times 2^{\text {exponent-127 }} \times 1$. mantissa

Elementary operations like addition and multiplication need normalization and de-normalization functions which use 48 bit barrel shifter for single precision floating point. This can cause poor fitting, slow clock rate and excessive routing. Hence floating point becomes a bottleneck in hardware implementations [6]. An alternative method that addresses this issue is the fused datapath methodology. Fused datapath integrates the basic operations into one single function and eliminate as many normalization and de-normalization step as possible [16-18].

\section{Simulation Results}

The design environment chosen in this paper is Simulink, which provides a high level of design description. It easily switches between fixed-point floating-point processing. The testbench used in the system-level simulation is later used to verify the FPGA-based implementation. The automated back-end synthesis tool running under Simulink is DSP Builder from Altera. Advanced Blockset (DSPBA) can provide a model based design based on fused datapath method. It also supports fixed point implementation by applying a set of libraries including fixed and floating point together with data type propagation. The proposed design is implemented in both fixed and floating point formats to compare the results.

The two main parameters are the matrix size and vector size. The first one is a design parameter defined according to the device resources and the latter is a user variable and may be of any size. The performance and resource usage of the QR decomposition have been evaluated for different matrix and vector sizes implemented on Stratix IV EP4SGX230KF40C2 and Arria 10AX115U5F45I3SP devices. The StratixIV device can accommodate vector sizes of up to 60 complex elements whereas the Arria10 device can accommodate vector sizes of up to 90 complex elements [14]. To demonstrate the effect of vector size on the QR decomposition performance, a 30 by 10 matrix is 
decomposed with different vector sizes for both fixed and floating point data types.

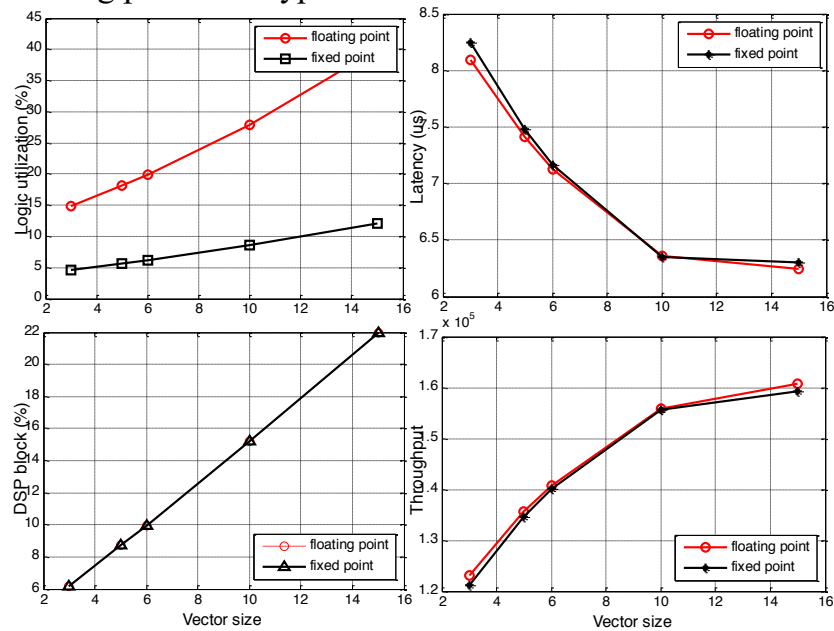

Fig. 5. Effect of vector size on a) resource utilization and b) performance for QR decomposition of a 30 by 10 matrix

Fig. 5a shows that the DSP blocks of StratixIV increases linearly with the vector size for both the fixed and floating point representations but the logic utilization increases significantly for the floating point architecture. Fig. 5b shows that by increasing the vector size latency decreases whereby the number of matrices processed per second, throughput, increases.

Fig. 6a shows the effect of matrix size on the resource utilization and Fig. $6 \mathrm{~b}$ shows its effect on performance for two FPGA implementations with the fixed vector size of 10 . As it is evident, large matrix sizes impose longer latencies and therefore lower throughput.

Arria10 is the first FPGA with hardened floating point DSP blocks which makes it suitable for floating point arithmetic. Fig. 7a shows comparison between StratixIV and Arria10 devices for different size matrices and different vector sizes for floating point data type.
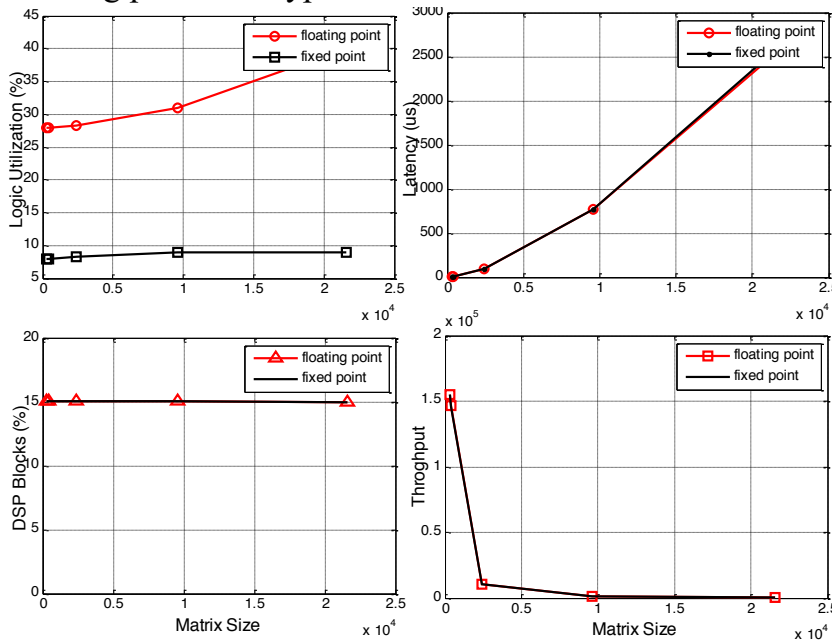

Fig. 6. Effect of matrix size on a) resource utilization and b) performance with a fixed vector size of 10

Arria10 uses five times less DSP blocks than StratixIV for the four combinations. Logic elements and memory bits usage are also decreased with nearly the same rate in Arria10. To show the number of DSP blocks, Memory bits and Logic elements in one graph for comparison they are normalized.

Fig. $7 \mathrm{~b}$ shows that in the fixed point implementations the Arria10 uses more DSP blocks as it should use the hardened floating point DSP blocks for fixed point data type. StratixIV uses nearly the same amount of the DSP blocks for both data types. Logic elements usage for floating point is 3-4 times higher than the fixed point for all matrix sizes and the two devices. Memory bits usage is not affected by the data type because both uses 32-bit representation. As expected the number of memory bits and Logic elements are increase by increasing the matrix size.
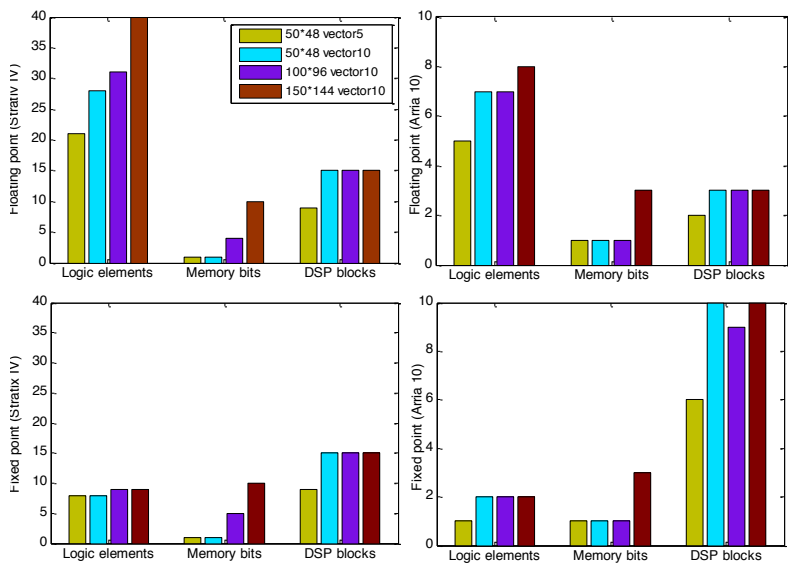

Fig. 7. a) StratixIV and b) Arria10 simulation results for fixed and floating point

To investigate the accuracy of the design, the calculated Q and $\mathrm{R}$ elements are compared to the double precision results obtained from the QR decomposition function in Matlab. Table1 shows the error performance. Frobenius Norm was used to measure the overall error magnitude in the resultant matrix:

$$
\|E\|_{F}=\sqrt{\sum_{i=1}^{N} \sum_{j=1}^{N}\left|e_{i j}\right|^{2}}
$$

Table1: Error performance

\begin{tabular}{cllcc}
\hline \hline Matrix size & \multicolumn{2}{c}{ Fixed point error } & \multicolumn{2}{c}{ Floating point error } \\
\hline & Norm & Max & Norm & Max \\
$50 * 48$ & 0.0402 & 0.0062 & 0.017 & 0.0031 \\
$100 * 96$ & 0.0533 & 0.0071 & 0.0328 & 0.0041 \\
$150 * 144$ & 0.0905 & 0.0115 & 0.0385 & 0.0081 \\
\hline \hline
\end{tabular}

The error analysis has been done on 3 different matrix sizes. The standard deviations are also included to show how far the elements of matrix $\mathrm{R}$ are spread out in each case. Results show that the fixed point implementation imposes more error than the floating point which was expected.

\section{HARDWARE IMPLEMENTATION AND COMPARISON}

In order to evaluate the performance of the proposed design in hardware, fixed and floating point QR core for a $4 \times 4$ matrix was implemented on the StratixIV FPGA. Table 2 shows the results in terms of latency, throughput, maximum clock frequency and the number of the clock cycles per each calculation alongside with the proposed architectures in [1923]. As can be seen, the throughput of the proposed design for both fixed and floating point implementations are better than the other architectures. With the higher word length the data accuracy of this design would be much higher although the data for other works are not available. 
TABLE 2: COMPARISON OF THE ACHIEVEMENTS OF THIS WORK WITH OTHER SIMILAR WORKS

\begin{tabular}{|c|c|c|c|c|c|c|c|}
\hline & [19] & {$[20]$} & {$[21]$} & {$[22]$} & {$[23]$} & \multirow{2}{*}{\multicolumn{2}{|c|}{$\begin{array}{c}\text { This work } \\
\text { Stratix- } \\
\text { Ep4SGX }\end{array}$}} \\
\hline Device & $\begin{array}{c}\text { Stratix } \\
\text { EP1S40 }\end{array}$ & $\begin{array}{c}\text { Stratix } \\
\text { EP1S40 }\end{array}$ & $\begin{array}{l}\text { XC2VP30- } \\
\text { Xilinx }\end{array}$ & $\begin{array}{l}\text { Virtex } 5 \\
\text { XUP }\end{array}$ & $\begin{array}{c}\text { Virtex4- } \\
\text { xc4vlx200 }\end{array}$ & & \\
\hline $\begin{array}{l}\text { Word } \\
\text { length }\end{array}$ & & & 20 & 16 & 21 & 32 & 32 \\
\hline Data Type & $\begin{array}{l}\text { Fixed } \\
\text { point }\end{array}$ & $\begin{array}{l}\text { Fixed } \\
\text { point }\end{array}$ & $\begin{array}{l}\text { Fixed } \\
\text { point }\end{array}$ & $\begin{array}{c}\text { Fixed } \\
\text { point }\end{array}$ & $\begin{array}{c}\text { Floating } \\
\text { point }\end{array}$ & $\begin{array}{l}\text { Fixed } \\
\text { point }\end{array}$ & $\begin{array}{c}\text { Floating } \\
\text { point }\end{array}$ \\
\hline $\begin{array}{l}\text { Number of } \\
\text { clock pulses }\end{array}$ & 73 & 54 & 294 & 180 & 933 & 142 & 170 \\
\hline Latency $(\mu \mathrm{s})$ & 0.97 & 0.9 & 1.3 & 0.73 & 6.6 & 0.42 & 0.49 \\
\hline $\begin{array}{c}\text { Max clock } \\
\text { Frequency } \\
(\mathrm{MHz})\end{array}$ & 75 & 60 & 48 & 246 & 115 & 340 & 360 \\
\hline $\begin{array}{c}\text { Throughput } \\
\text { (M/sec) }\end{array}$ & 1.03 & 1.11 & 0.72 & 1.36 & 0.15 & 2.4 & 2.11 \\
\hline
\end{tabular}

\section{CONCLUSION}

In this paper an efficient FPGA implementation of QR decomposition is presented. The decomposition core was first designed in DSP Builder Advanced Blockset and then the VHDL code was synthetized in Quartus II software and finally it was implemented on Stratix Ep4SGX230KF40C2 device. For a $4 \times 4$ matrix, throughputs of $2.4 \mathrm{M}$ and $2.11 \mathrm{M}$ matrix per second are achieved for fixed and floating point respectively. The results shows that although fixed point design utilizes less FPGA resources but it suffers from less accuracy compared to floating point probably due to quantization and round off errors. It was also shown that there must be a trade-off between vector and matrix size in order to achieve the best performance and less area.

\section{REFERENCES}

[1] M. Mahmoodi, B. M. Abadi, H. Khajepur, and M. H. Harirchian. "A robust beamforming approach for early detection of readiness potential with application to brain-computer interface systems." In 2017 39th Annual International Conference of the IEEE Engineering in Medicine and Biology Society (EMBC), pp. 2980-2983. IEEE, 2017.

[2] S. Amin-Nejad, T. A. Gashteroodkhani, K. Basharkhah, "A Comparison of MVDR and LCMV Beamformers' Floating Point Implementations on FPGAs," Wireless Personal Communications, vol.98, no. 2 , pp.1913-1929, 2018.

Available: https://link.springer.com/article/10.1007/s11277-017-4953$\underline{1}$

[3] A. El-Amawy and K. R. Dharmarajan, "Parallel VLSI algorithm for stable inversion of dense matrices", IEEE Proceedings on Computers and Digital Techniques, Volume 136, Issue 6, , Nov 1989, pp.575-580.

[4] M. Mirmozaffari, "Eco-Efficiency Evaluation in Two-Stage Network Structure: Case Study: Cement Companies". Iranian Journal of Optimization (IJO). Dec. 16, 2018.

[5] P. N. Ganchosov, G.K. Kuzmanov, H. Kabakchiev, V. Behar, R P.Romansky, G. N. Gaydadjiev, "FPGA Implementation of Modified Gram-Schmidt QR-Decomposition", Proceedings of the 3rd HiPEAC Workshop on Reconfigurable Computing, Paphos, Cyprus, Jan. 2009, pp. 41-51.

[6] Michael Parker and Dan Pritsker "Floating Point Vector Processing using 28nm FPGAs", Proceedings of the 2012 HPEC conference, September 2012

[7] Ali Irturk, Shahnam Mirzaei and Ryan Kastner, "An Efficient FPGA Implementation of Scalable Matrix Inversion Core using QR Decomposition", UCSD Technical Report, CS2009-0938, 2009.

[8] P. Luethi, C. Studer, S. Duetsch, E. Zgraggen, H. Kaeslin, N. Felber, and W. Fichtner, "Gram-schmidt-based QR decomposition for MIMO detection: VLSI implementation and comparison," in IEEE Asia Pacific Conference on Circuits and Systems, APCCAS, 2008, pp.830833.

[9] Richard L. Walke, Robert W. M. Smith and Gaye Lightbody,"20GFLOPS QR processor on a Xilinx Virtex-E FPGA", International Society for Optics and Photonics. Proceeding of SPIE Advanced Signal Processing Algorithms, Architectures and implementations X, 2000, vol.4116, pp. 300-310.

[10] H. Firouzkouhi, "FPGA Based Implementation of Cascaded Multilevel Inverter with Adjustable DC Sources", International Journal of Research and Engineering (IJRE), vol. 5, no. 7, pp. 450-456, July 2018. Available: https://hal.archives-ouvertes.fr/hal-02182656/document

[11] I. Berkeley Design Technology, “An Independent Analysis of Altera's FPGA Floating-point DSP Design Flow," 2011.

[12] M. Mirmozaffari, A. Alinezhad, "Ranking of Heart Hospitals Using cross-efficiency and two-stage DEA," 7th International Conference on Computer and Knowledge Engineering (ICCKE), Mashhad, pp. 217222, 2017.

[13] A. Forooghi Nematollahi, A. Dadkhah, O. Asgari Gashteroodkhani, and B. Vahidi, "Optimal sizing and siting of DGs for loss reduction using an iterative-analytical method," Journal of Renewable and Sustainable Energy, vol. 8, p. 055301, 2016.

[14] Michael Parker and Volker Mauer, "Floating Point STAP Implementation on FPGAs", Radar Conference (RADAR), IEEE, May 2011, pp. 901-904.

[15] Chitranjan K. Singh, Sushma Honnavara Prasad, and Poras T. Balsara," A Fixed-Point Implementation for QR Decomposition", proceeding of IEEE workshop on Design, Applications, Integration and Software, October 2006, pp75-78.

[16] Martin Langhammer, Tom VanCourt, "FPGA Floating Point Compiler", Proceedings of the 2009 17th IEEE Symposium on Field Programmable Custom Computing Machines, pp259-262, 2009

[17] M. Langhammer, "High performance matrix multiply using fused datapath operators," in 2008 42nd Asilomar Conference on Signals, Systems and Computers. IEEE, October 2008, pp. 153-159

[18] Suleyman S. Demirsoy and Martin Langhammer, "Fused Datapath Floating Point Implementation of Cholesky Decomposition," Proceedings of the ACM/SIGDA International Symposium on Field Programmable Gate Arrays, February 22 - 24, 2009

[19] Y. Y. K. M. A. Hiroyuki, "Resource and performance evaluations of fixed point QRD-RLS systolic array through FPGA implementation, IEICE Trans. on Communications" [Online] (4) (2008) 1068-1075.

[20] D. Chen and M. Sima, "Fixed-point CORDIC-based QR decomposition by Givens rotations on FPGA," International Conference on Reconfigurable Computing and FPGAs, 2011, Nov 2011, pp. 327-332.

[21] Boonyi, K, Tagapanij, J and Boonpoonga, A., "FPGA-based Hardware/Software Implementation for MIMO Wireless Communications", International Electrical Engineering Congress (iEECON), March 2014, pp. 1-4.

[22] S. Aslan, S. Niu, and J. Saniie, "FPGA implementation of fast QR decomposition based on Givens rotation," in, IEEE 55th International Midwest Symposium on Circuits and Systems, 2012, pp. 470-473.

[23] Karkooti, M, Cavallaro, J.R, Dick, C., "FPGA Implementation of Matrix Inversion Using QRD-RLS Algorithm", Thirty-Ninth Asilomar Conference on Signals, Systems and Computers, October 2005, pp. 1625-1629.

\section{APPENDIX}

Appendix 1:

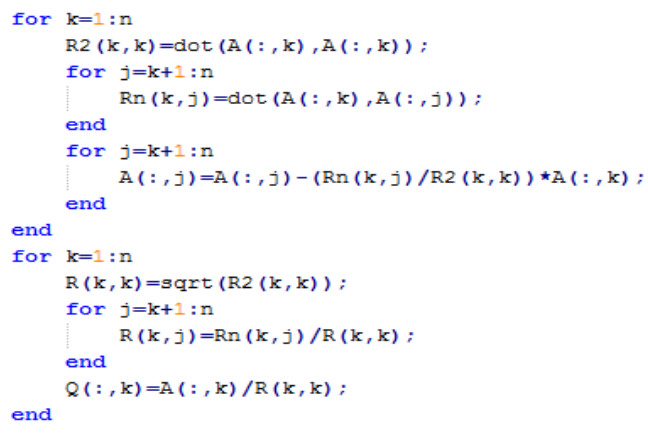

Appendix 2:

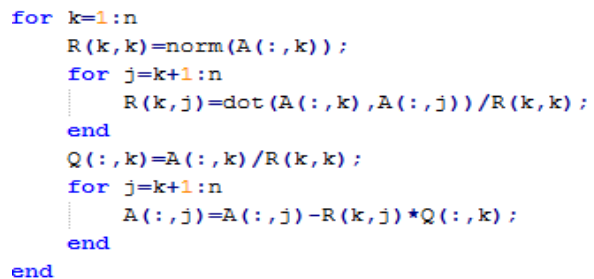

